
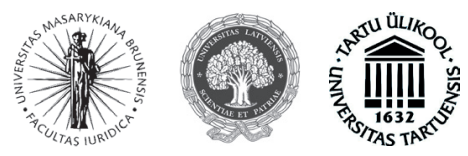

ISSN 1392-6195 (print) ISSN 2029-2058 (online) JURISPRUDENCIJA JURISPRUDENCE 2013, 20(3), p. 901-922.

\title{
DERECHOS DE LAS MINORÍAS EN EL PACTO INTERNACIONAL DE DERECHOS CIVILES Y POLÍTICOS: CONSIDERACIONES CONCEPTUALES
}

\author{
Fernando Arlettaz \\ Doctor en Sociología Jurídica e Instituciones Políticas \\ Investigador en el Laboratorio de Sociología Jurídica de la Universidad de Zaragoza \\ C/Pedro Cerbuna 12 - 50009 Zaragoza - España \\ Telephone: 0034976761353 \\ E-mail: arlettaz@unizar.es
}

Fecha de envío: 22 de junio de 2013.

doi:10.13165/JUR-13-20-3-04

Resumen. La protección de las minorias, en el sentido amplio del término, implica tanto la garantía de la igualdad formal y sustantiva en relación con ellas como la preservación de sus características identitarias propias. El artículo establece una distinción conceptual entre derechos universales, derechos especificos de las minorias en general y derechos especificos de minorias determinadas. Se analiza el Pacto Internacional de Derechos Civiles y Politicos: por un lado, el artículo 27 que establece derechos específicos de las minorias en general con la finalidad de proteger y promover sus formas de vida; por otro lado, los artículos 2.1 y 26 que establecen un derecho universal a la no discriminación que puede ser invocado por las minorias. En ambos casos, la transformación de los derechos reconocidos en términos amplios por el Pacto en derechos más concretos por medio de medidas positivas puede dar lugar al nacimiento de derechos especificos de minorias determinadas.

Palabras clave: minorias, Pacto Internacional de Derechos Civiles y Politicos, Comité de Derechos Humanos, discriminación, medidas positivas. 


\section{Introducción}

La cuestión de las minorías tiene una conocida historia en el campo del derecho internacional público ${ }^{1}$. Desde las guerras de religión en el comienzo de la modernidad, un problema crucial de la politica internacional, que ha tenido su reflejo en el derecho internacional, ha sido el de la situación de las minorías nacionales, religiosas y lingüísticas. Luego de la anexión de territorios como consecuencia de las guerras religiosas de comienzos de la modernidad las potencias europeas firmaron diferentes tratados protegiendo a las minorías que residían en esos territorios.

Luego de la primera guerra mundial se reforzaron los mecanismos de protección de las minorías. Sin embargo, estos mecanismos, establecidos a partir de tratados bilaterales y multilaterales, no reconocieron directamente derechos de los individuos y de los grupos, sino que impusieron obligaciones a los Estados, en cuestiones de acceso a cargos públicos, libertad en el uso de la lengua, educación, etc. Estos tratados, de redacción compleja, tenían un doble objetivo: por un lado, asegurar la no discriminación de los nacionales que pertenecían a minorías en el ejercicio de los derechos civiles y políticos comunes; por otro lado, garantizar a las personas pertenecientes a las minorías un conjunto de derechos especiales que debía permitirles preservar su cultura y formas de vida. Se constituyó de este modo un sistema de minorías en el marco de la Sociedad de Naciones. La Corte Permanente de Justicia Internacional desarrolló una importante jurisprudencia en este sentido ${ }^{2}$, en la que se aceptaba la doble caracterización de los derechos de las minorías recién señalada.

Luego de la Segunda Guerra Mundial, y en razón del uso abusivo que Alemania había hecho de las cláusulas de protección de sus minorías en el extranjero, la atención se orientó hacia la protección universalista de los derechos humanos, con más énfasis en la igualdad que en la protección de las particularidades minoritarias. De hecho, en los primeros años de vida del sistema de Naciones Unidas, se pensaba que mediante el reconocimiento de derechos universales podría lograrse la desactivación de las reclamaciones específicas de las minorías ${ }^{3}$. Esto no impidió, sin embargo, que las minorías recibieran un tratamiento específico en el artículo 27 del Pacto Internacional de Derechos Civiles y Políticos (a partir de ahora, el Pacto) ${ }^{4}$. Más recientemente, la

1 Para la evolución histórica, ver Lerner, N. The Evolution of Minority Rights in International Law. Peoples and Minorities in International Law. London: Martinus Nijhoff Publishers, 1993, p. 77-101. Rouland, N.; Pierré-Caps, S.; Poumarède, J. Droit des minorités et des peuples autochtones. Paris: Presses Universitaires de France, 1996. Díaz Pérez de Madrid, A. La protección de las minorías en Derecho internacional. Granada: Editorial Universidad de Granada, 2004. Thio, L. Managing Babel: The International Legal Protection of Minorities in the Twentieth Century. The Hague: Martinus Nijhoff Publishers, 2005.

2 Entre los casos más importantes se encuentran Rights of Minorities in Upper Silesia (Minority Schools), Germany v. Poland (Judgment), 1928 PCIJ Series A, 15; Access to German minority schools in Upper Silesia (Advisory Opinion), 1931 PCIJ Series A/B, 40; Minority Schools in Albania (Advisory Opinion), 1935 PCIJ Series A/B 64.

3 Pentassuglia, G. Minority Groups and Judicial Discourse in International Law, The Hague: Martinus Nijhoff Publishers, 2009, p. 3.

4 Pacto Internacional de Derechos Civiles y Políticos (adoptado 16/12/1966, en vigor desde 23/03/1976). UNTS 1-14668. 
Asamblea General de las Naciones Unidas consideró el tema a través de la Declaración sobre los Derechos de las Personas pertenecientes a Minorías Nacionales o Étnicas, Religiosas y Lingüísticas (a partir de ahora, la Declaración) ${ }^{5}$. En el ámbito de las Naciones Unidas hay que mencionar además la Declaración de la UNESCO sobre la diversidad cultural ${ }^{6}$. La cuestión de las minorias también ha sido objeto de atención en los sistemas regionales de protección de los derechos humanos ${ }^{7}$.

El propósito de este trabajo es realizar un estudio teórico sobre la configuración estructural de los derechos de las minorías en el Pacto. No se trata de analizar con detalle cada uno de los derechos reconocidos y los precedentes internacionales que pudieran existir en relación con ellos, sino de abordar la temática desde la perspectiva de las diferentes categorías de derechos y sus relaciones recíprocas. El estudio específico de los derechos de las minorías a la luz del Pacto ha sido realizado en otros trabajos ${ }^{8}$, en los que nosotros nos apoyaremos para discutir las categorías teóricas generales que aquí nos interesan.

Para realizar esta tarea la metodología empleada será la de la ciencia jurídica, con las particularidades propias del derecho internacional de los derechos humanos. Aunque

5 Aprobada por la Asamblea General de las Naciones Unidas (Resolución 47/135 de 18/12/1992). Ver Lerner, N. Las Naciones Unidas y las minorías: a propósito de la Declaración de la Asamblea General de 18 de diciembre de 1992. Revista española de derecho internacional. 1993, 45(2): 269-282. También Commentary on the United Nations Minorities Declaration, Working Group on Minorities, E/CN.4/ Sub.2/AC.5/2005/2.

6 Declaración Universal de la UNESCO sobre la Diversidad Cultural (02/11/2001).

7 Un problema previo, al que no nos referiremos aquí, es el de la definición del concepto de minoría. Ver Andrysek, O. Report on the Definition of Minorities. Netherlands: Netherlands Institute of Human Rights, 1986. Bossuyt, M. The United Nations and the Definition of Minorities. Plural Societes. 1990, 21: 129-136. Ramaga, P. The Group Concept in Minority Protection. Human Rights Quarterly. 1993, 15(3): 575-588. Rodley, N. Conceptual Problems in the Protection of Minorities: International Legal Development. Human Rights Quarterly. 1995, 17(1): 48-71. González Hidalgo, E.; Ruiz Vieytez, E. La definición implícita del concepto de minoría nacional en el Derecho Internacional. Derechos y libertades: Revista del Instituto Bartolomé de las Casas. 2012, 27: 17-56.

8 Ermacora, F. The protection of minorities before the United Nations, Recueil des Cours 182. Leiden: Martinus Nijhoff Publishers, 1983. P. Thornberry. International Law and the Rights of Minorities. Oxford: Clarendon Press, 1991. Capotorti, F. Study of the Rights of Persons belonging to Ethnic, Religious and Linguistic Minorities. New York: United Nations, 1991. Eide, A. Protection of minorities: possible ways and means of facilitating the peaceful and constructive solution of problems involving minorities, E/CN.4/ Sub.2/1993/34. Henrard, K. Devising an Adequate System of Minority Protection. Individual Human Rights, Minority Rights and the Right to Self-Determination. The Hague: Martinus Nijhoff Publishers, 2000. Díaz Pérez de Madrid, A. La protección de las minorías en Derecho internacional. Petričušić, A. The Rights of Minorities in International Law: Tracing Developments in Normative Arrangements of International Organizations. Croatian International Relations Review, 2005, XI(38/39): 7. Pentassuglia, G. Minorities in international law: an introductory study. Strasbourg: Council of Europe Publications, 2002. Vijapur, A. International protection of minority rights. International Studies, 2006, 43(4): 367-394. Peter Hilpold, UN Standard-setting in the field of minority rights. International Journal on Minority and Group Rights, 2007, 14: 181-205. Kymlicka, W. The Internationalization of Minority Rights. International Journal of Constitutional Law, 2008, 6(1): 1-32. Pentassuglia, G. Minority Groups and Judicial Discourse in International Law. Henrard, K. Minorities, International Protection. Max Planck Encyclopedia of Public International Law [interactivo]. 2010 [visitado 20/08/2013]. http://www.mpepil. com. Barelli, M. et. al. Minority groups and litigation: A review of developments in international and regional jurisprudence. Liverpool: Minority Rights Group International, 2011. 
el objetivo central es el estudio del Pacto, la Declaración servirá como un punto de apoyo interpretativo. Se consultarán asimismo los informes preparados por encargo de la Organización de las Naciones Unidas, los precedentes del Comité de Derechos Humanos y la opinión de la doctrina especializada.

Resulta ante todo necesario establecer ciertas distinciones en el plano puramente conceptual, es decir, prescindiendo del contenido concreto de cualquier derecho positivo. Una primera distinción es la que corresponde realizar entre derechos universales, derechos especificos de las minorias en general y derechos especificos de minorias determinadas ${ }^{9}$. Los primeros son aquellos que corresponden a todos los individuos (por ejemplo, "toda persona tiene derecho a no ser torturada") o a todos los grupos de una determinada clase (por ejemplo, "todas las familias tienen derecho a tal protección"). Los grupos minoritarios y sus miembros son titulares de estos derechos en la misma medida que cualquier otro individuo o grupo. Los derechos específicos de las minorías en general, en cambio, son aquellos que se conceden a los grupos minoritarios en sí mismos ("todos los grupos minoritarios tienen derecho al uso de su lengua") o a sus miembros ("todos los individuos pertenecientes a una minoría tienen derecho al uso de la lengua minoritaria"). Finalmente, hay algunos derechos específicos de grupos minoritarios determinados ("el grupo minoritario $\mathrm{X}$ tiene derecho al uso de la lengua $\mathrm{Y}$ ") o de los miembros de grupos minoritarios determinados ("los miembros del grupo minoritario X tienen derecho al uso de la lengua Y").

Los instrumentos del derecho internacional de los derechos humanos suelen reconocer derechos universales y derechos específicos de las minorías en general. Sin embargo, la implementación de estos derechos, mediante medidas positivas de parte del Estado que hagan que los derechos reconocidos en términos amplios asuman una forma más concreta, llevará en muchos casos al nacimiento de derechos específicos de grupos minoritarios determinados o de derechos específicos de los individuos pertenecientes a grupos minoritarios determinados.

Hay que tener en cuenta, como ha señalado Henrard ${ }^{10}$ retomando el criterio de la Corte Permanente de Justicia Internacional, que la protección de las minorías (en el sentido amplio de la expresión) se asienta en dos pilares: por un lado, la protección contra la discriminación; por otro lado, la protección de las minorías en sentido estricto. El primer pilar es una manifestación del principio general de no discriminación, y exige tanto el respeto a la igualdad formal respecto de las minorías como la garantía de la igualdad sustantiva. La protección de las minorías en sentido estricto implica proteger

9 Una distinción parcialmente semejante es la que ha sido realizada entre normas de protección específicas de las minorías y normas de protección no específicas de las minorías. Henrard, K. Minorities, International Protection, p. 7. Por nuestra parte preferimos la distinción triádica que formulamos en el cuerpo del texto.

10 Henrard, K. Devising an Adequate System of Minority Protection. Individual Human Rights, Minority Rights and the Right to Self-Determination, p. 8-11. Henrard, K., Minorities, International Protection, p. 4. Henrard, K. The Interrelationship between Individual Human Rights, Minority Rights and the Right to Self-Determination and Its Importance for the Adequate Protection of Linguistic Minorities. The Global Review of Ethnopolitics. 2001, 1(1): 43-44. 
la identidad específica de éstas. El primer pilar es un requisito para el desarrollo del segundo, que asume la existencia del primero y se construye sobre él.

La finalidad de la igualdad en sentido formal se logra mediante la titularidad, por parte de los grupos minoritarios y de sus miembros, de los mismos derechos de los que son titulares otros grupos e individuos. Para alcanzar la igualdad en sentido sustantivo, en cambio, es posible que resulten necesarias medidas positivas. La implementación de estas medidas positivas se hará normalmente mediante derechos específicos de los grupos minoritarios o de sus miembros. Finalmente, la protección de las características distintivas de las minorías se hará también mediante derechos específicos.

De este modo, existen derechos específicos de las minorías que tienen por finalidad la protección de la diferencia y derechos específicos que tienen por finalidad la protección de la igualdad sustantiva. Los primeros buscan proteger y promover las particularidades propias de la cultura minoritaria, como es el caso por ejemplo del derecho a recibir educación en la propia lengua. Los segundos, en cambio, están reconocidos para superar la situación de desventaja en la que pueden encontrarse los grupos minoritarios o sus miembros fomentando un acceso igualitario a los recursos y las oportunidades sociales. Piénsese por ejemplo en la llamada discriminación inversa que ha sido aplicada para favorecer el acceso de los miembros de ciertos grupos étnicos a las universidades estadounidenses. No se trata en este caso de proteger las peculiaridades culturales, religiosas o lingüísticas de un grupo, sino por el contrario de brindar a ese grupo y a sus miembros condiciones más favorables para acceder a un recurso social que tiene carácter uniforme (en este caso, la educación universitaria).

En el apartado 1 de este trabajo nos referiremos al artículo 27 del Pacto que reconoce derechos específicos de los individuos pertenecientes a grupos minoritarios en general, al establecer que "en los Estados en que existan minorías étnicas, religiosas o lingüísticas, no se negará a las personas que pertenezcan a dichas minorías" los derechos que luego se enumeran. En el apartado 2 nos referiremos a los artículos 2.1 y 26 del Pacto, que reconocen el principio de igualdad y no discriminación. En el apartado 3 analizaremos las medidas positivas que pueden ser necesarias para la concreta implementación de estos artículos.

\section{El artículo 27 del Pacto}

El artículo 27 del Pacto Internacional de Derechos Civiles y Políticos busca la protección y promoción de las formas de vida de las minorías mediante el reconocimiento de derechos específicos en favor de los individuos pertenecientes a las minorías. El Comité de Derechos Humanos ha señalado que estos derechos son diferentes de los derechos consagrados en otros artículos del Pacto ${ }^{11}$. Lo mismo puede decirse de la Declaración

11 General Comment No. 23: The rights of minorities (Art. 27), CCPR/C/21/Rev.1/Add.5, párrafo 1. Se debe distinguir, por ejemplo, el derecho del artículo 27 a usar el propio idioma por parte de los miembros de la minoría de otros derechos como la libertad de expresión del artículo 19 o el derecho del acusado de tener un traductor cuando no puede entender o hablar el idioma del tribunal del artículo 14.3.f (General Comment No. 23: The rights of minorities (Art. 27), párrafo 5.3). 
sobre los derechos de las personas pertenecientes a minorías, que establece que los derechos por ella reconocidos lo son "sin perjuicio del disfrute por todas las personas de los derechos humanos y las libertades fundamentales reconocidos universalmente” (artículo 8.2).

El artículo 27 se refiere, en términos amplios, a tres derechos de los individuos pertenecientes a grupos minoritarios. Se trata de los derechos a "tener su propia vida cultural, a profesar y practicar su propia religión y a emplear su propio idioma". La Declaración, por su parte, contiene 9 artículos en los que se establecen derechos de los individuos pertenecientes a minorías y obligaciones de los Estados. Lo fundamental de la Declaración está enunciado en su artículo 1.1: "Los Estados protegerán la existencia y la identidad nacional o étnica, cultural, religiosa y lingüística de las minorías dentro de sus territorios respectivos y fomentarán las condiciones para la promoción de esa identidad". El artículo 2, por su parte, reconoce los principales derechos de los individuos pertenecientes a minorias: "1. (...) derecho a disfrutar de su propia cultura, a profesar y practicar su propia religión, y a utilizar su propio idioma, en privado y en público, libremente y sin injerencia ni discriminación de ningún tipo. 2. (...) derecho de participar efectivamente en la vida cultural, religiosa, social, económica y pública. 3. (...) derecho de participar efectivamente en las decisiones que se adopten a nivel nacional y, cuando proceda, a nivel regional respecto de la minoría a la que pertenezcan o de las regiones en que vivan, de toda manera que no sea incompatible con la legislación nacional. 4. (...) derecho de establecer y mantener sus propias asociaciones. 5. (...) derecho a establecer y mantener, sin discriminación de ningún tipo, contactos libres y pacíficos con otros miembros de su grupo y con personas pertenecientes a otras minorías, así como contactos transfronterizos con ciudadanos de otros Estados con los que estén relacionados por vínculos nacionales o étnicos, religiosos o lingüísticos" $"$.

Aunque el artículo 27 no se refiera directamente a derechos económicos, los derechos en él reconocidos pueden tener una dimensión económica. Así, en el caso Ominayak v. Canadá, una comunidad aborigen (la comunidad del Lago Lubicon) representada por su

12 La Declaración establece la obligación de los Estados de proteger no sólo la identidad nacional, étnica, religiosa o lingüística de las minorías, sino también su identidad cultural (a pesar de que el título de la Declaración se refiere a minorías nacionales o étnicas, religiosas y lingüísticas, pero no minorías culturales). Además de los derechos expresamente reconocidos, la Declaración se refiere a una serie de obligaciones generales de los Estados. En primer lugar, la de tener debidamente en cuenta los intereses legítimos de las personas pertenecientes a minorías en la planificación y ejecución de sus políticas, así como en la cooperación interestatal (artículo 5). En segundo lugar, la de cooperar en las cuestiones relativas a personas pertenecientes a minorías (artículo 6) y para promover el respeto de los derechos enunciados en la Declaración (artículo 7). Finalmente, se aclara que ninguna de las disposiciones de la Declaración impide el cumplimiento de otras obligaciones internacionales por parte de los Estados en relación con las personas pertenecientes a minorías (artículo 8.1) y que los organismos especializados y demás organizaciones del sistema de las Naciones Unidas deben contribuir a la plena realización de los derechos y principios enunciados en ella (artículo 9).

Según ha dicho el Comité de Derechos Humanos, la protección de las formas de vida de las minorías no implica sólo el resguardo de sus formas tradicionales de vida, sino también de la posibilidad de adaptarlas a las condiciones de las sociedades modernas. Apirana Mahuika et al. v. Nueva Zelanda, communication 547/1993, CCPR/C/70/D/547/1993 (2000), párrafo 9.4. Ilmari Lansman et al. v. Finlandia, communication 511/1992, CCPR/C/52/D/511/1992 (1994), párrafo 9.3. 
jefe se quejaba de la expropiación dispuesta por el gobierno de Canadá sobre sus tierras tradicionales en las que practicaban la caza y la pesca. El Comité halló una violación al artículo 27 y sostuvo que "los derechos protegidos por el artículo 27 incluyen el derecho de las personas, en comunidad con otras, a realizar actividades económicas y sociales que son parte de la cultura de la comunidad a la que pertenecen"13.

No hay ninguna duda respecto del carácter vinculante de las obligaciones emergentes del artículo 27 del Pacto, que aunque está redactado en términos negativos ("[...] no se negará $[\ldots]$ '), reconoce la existencia de verdaderos derechos, de modo que el Estado está obligado a asegurarlos ${ }^{14}$. Es más: como afirma Pentassuglia, el artículo 27, al menos en sus aspectos más esenciales, parece estar en la base de un consenso que apunta a la formación de una costumbre internacional ${ }^{15}$. Respecto de la Declaración, en cambio, sólo puede afirmarse que se trata de una norma de soft-law.

En el plano conceptual, los derechos de las minorías pueden ser tanto de titularidad individual como colectiva. Sin embargo, tanto el Pacto como la Declaración se refieren no a los derechos de los grupos minoritarios sino a los derechos de los individuos pertenecientes a las minorías, y esto sin perjuicio de que tales derechos puedan ejercerse "en común con los demás miembros de su grupo". La cuestión del carácter individual o colectivo de los derechos emergentes del artículo 27 es de gran significación. Piénsese en la propiedad colectiva de la tierra por parte de grupos aborígenes, el ejercicio colectivo de derechos lingüísticos por parte de minorías territorialmente concentradas o el debate más contemporáneo sobre posibilidad de la titularidad colectiva de derechos por parte de las minorías religiosas surgidas de la inmigración.

Es difícil calificar adecuadamente los derechos emergentes del artículo 27 ya que, como se ha afirmado, su finalidad es la protección de los grupos minoritarios, pero saber si esta finalidad se traduce en la existencia de derechos colectivos depende en buena medida de la definición que se adopte acerca del concepto mismo de derecho ${ }^{16}$. Por nuestra parte creemos que, desde una perspectiva de estricta teoría jurídica, hay que decir que el artículo 27 sólo reconoce derechos individuales ${ }^{17}$. Las consecuencias reales de este reconocimiento, en cambio, pueden ser un poco más ambiguas. En primer lugar, porque uno de los derechos reconocidos a los individuos pertenecientes a grupos minoritarios es el derecho a formar asociaciones (artículo 2.4 de la Declaración). Así, el Comité ha sostenido que no existe obstáculo para que un grupo de personas

13 Bernard Ominayak Chef of the Lubicon Lake Band v. Canada, communication 167/1984, Supp. 40 (A/45/40) at 1 (1990), párrafo 32.2.

14 General Comment No. 23: The rights of minorities (Art. 27), párrafo 6.1.

15 Pentassuglia, G. Minority Rights, Human Rights: A review of basic concepts, entitlements and implementation procedures under International law. Mechanisms for the implementation of minority rights, Berlin: Council of Europe Publishing, 2004, p. 14.

16 Bisaz, C. The Concept of Group Rights in International Law. Leiden: Martinus Nijhoff Publishers, 2012, p. 110-119.

17 Ver esta posición en Henrard, K., Minorities, International Protection, p. 10. Meijknecht, A. Towards International Personality: The Position of Minorities and Indigenous Peoples in International Law. Antwerpen: Intersentia, 2001, p. 136. 
pertenecientes a un grupo minoritario que alegan haber sufrido violaciones semejantes se presenten conjuntamente ante él ${ }^{18}$.

En segundo lugar, porque aunque el Pacto y la Declaración configuran los derechos como derechos individuales, no impiden que puedan reconocerse derechos colectivos como forma de implementar tales derechos individuales. Por ejemplo, el Comité de Derechos Humanos admitió que era legítimo reconocer a los grupos minoritarios el derecho a decidir quiénes eran sus miembros. En el caso Kitok v. Suecia se analizó la situación de una persona que había sido excluida de la comunidad indígena Sami. Esta comunidad estaba tradicionalmente dedicada a la cría de renos, y según la legislación podía excluir de su seno a aquellos miembros que abandonasen esta actividad. El Comité entendió que el objetivo de la legislación era legítimo (asegurar la continuidad de la forma tradicional de vida así como garantizar la cría de renos) y consideró que también la aplicación en el caso había sido legítima ${ }^{19}$.

La oposición entre el interés de la minoría y el interés de sus miembros fue también abordado, más recientemente, en un caso relativo al derecho de pesca de los maoríes (Apirana Mahuika v. Nueva Zelanda). A finales del siglo XX los maoríes concluyeron un acuerdo que introducía un sistema de cuotas y que suponía una renuncia a sus pretensiones en todas las controversias que tenían pendientes con el Estado. Sin embargo, un grupo de miembros de la tribu maorí no aceptó el acuerdo. El Comité entendió que en el proceso de negociación del acuerdo se había prestado la necesaria atención al significado cultural y religioso de la pesca para los maoríes, de modo que el interés colectivo había sido salvaguardado. En cuanto al interés individual de los maoríes disidentes, el Comité sostuvo que mientras la aplicación del acuerdo permitiera que los disidentes siguieran gozando de su cultura y practicando la religión con los

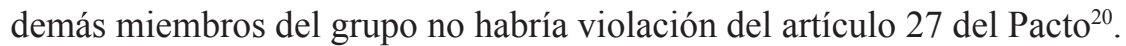

Sin embargo, las potestades de los grupos no son ilimitadas, como tuvo oportunidad de señalar el Comité en el caso Lovelace v. Canada, el primero abordado en relación con el artículo 27. El caso se refería a una mujer indígena que, tras haber contraído matrimonio con un no-indígena, había perdido según la legislación su derecho a residir en el territorio de su comunidad. El objetivo declarado de la prohibición era proteger la integridad de las comunidades indígenas, en particular en relación con sus tierras. El Comité juzgó que los objetivos de la legislación eran coherentes con el Pacto, pero dijo también que las medidas empleadas para estos fines debían "tener una justificación razonable y objetiva y ser compatibles con las demás disposiciones del Pacto"21. Dado que esto último no se producía, el Comité declaró la existencia de una violación del artículo 27.

Los derechos de las minorías no son absolutos. Nada impide que los Estados regulen diferentes actividades que constituyan partes esenciales de las formas de vida

18 Bernard Ominayak Chef of the Lubicon Lake Band v. Canada, párrafo 32.1.

19 Ivan Kitok v. Sweden, communication 197/1985, CCPR/C/33/D/197/1985 (1988).

20 Apirana Mahuika et al. v. New Zealand.

21 Sandra Lovelace v. Canada, communication R.6/24, Supp. No. 40 (A/36/40) at 166 (1981), párrafo 7. 
de las minorías, con tal que esta regulación no equivalga a una denegación de facto de los derechos que les han sido internacionalmente reconocidos. Además, aquellos actos que tengan una escasa trascendencia en la forma de vida de las minorías, y que por ello no impliquen una gran interferencia con ella, no significan una violación de los derechos de éstas.

En el caso Ilmari Länsman et al. v. Finlandia el Comité analizó la denuncia de un grupo de miembros de la comunidad Sami que alegaba que la decisión del Estado de permitir la excavación y el transporte de roca por sus tierras tradicionales, en las que practicaban la cría de renos como parte de su forma tradicional de vida, violaba el artículo 27. El Comité concluyó que se trataba de una decisión estatal con escasa trascendencia en las prácticas tradicionales, y que por eso no había violación del artículo 27 del Pacto ${ }^{22}$. En el caso Jouni Länsman et al. v. Finlandia se llegó a la misma conclusión respecto de las actividades de explotación forestal y la construcción de una carretera en el territorio tradicionalmente dedicado por los Sami a la cría de renos ${ }^{23}$. Sin embargo, el Comité dejó abierta la posibilidad de que si la explotación crecía al punto de afectar su modo de vida, la comunidad Sami pudiera volver a presentar el caso. Esto sucedió años más tarde: el Comité volvió a examinar la situación teniendo en cuenta la ampliación del área de explotación forestal, pero nuevamente concluyó que la incidencia en la forma de vida de la comunidad no era significativa y por lo tanto no había violación del artículo 27 del Pacto $^{24}$. En el caso Howard v. Canadá ${ }^{25}$ se llegó a la conclusión de que el hecho de que el Estado exigiera una licencia para que los miembros de una comunidad aborigen pescaran fuera de su reserva no implicaba por sí una violación de los derechos del artículo 27.

Resulta de interés referirse a la situación de los extranjeros. Las migraciones de la era de la globalización están dando lugar a la aparición de nuevas minorías, cuyas características son diferentes de las de las minorías nacionales surgidas de la definición de las fronteras del Estado nación, de las minorías surgidas de la fijación de fronteras poscoloniales o de los grupos aborígenes ${ }^{26}$. Según el Comité, los derechos reconocidos en el artículo 27 protegen tanto a nacionales como a extranjeros, siempre por supuesto que estos últimos reúnan los requisitos necesarios para ser considerados una minoría ${ }^{27}$.

22 Ilmari Lansman et al. v. Finlandia.

23 Jouni E. Länsman et al. v. Finland, communication 671/1995, CCPR/C/58/D/671/1995 (1996).

24 Jouni Länsman, Eino Länsman and the Muotkatunturi Herdsmen's Committee v. Finland, communication 1023/2001, CCPR/C/83/D/1023/2001 (2005). En otro caso, en el que sentencias judiciales habían denegado la propiedad de las tierras en las que los Sami practican su actividad tradicional, el Comité declaró que la comunicación era inadmisible porque no se habían agotado los recursos internos. Jarle Jonassen and members of the Riast/Hylling reindeer herding district v. Norway, communication 942/2000, CCPR/ $\mathrm{C} / 76 / \mathrm{D} / 881 / 1999$ (2002).

25 George Howard v. Canada, communication 879/1999, CCPR/C/84/D/879/1999 (2005).

26 Bengoa, J. Existence and recognition of minorities, working paper for the Working Group of Minorities, E/CN.4/Sub.2/AC.5/2000/WP.2, párrafos 23-24 y 69-75.

27 General Comment No. 15: The position of aliens under the Covenant, HRI/GEN/1/Rev.1 at 18, párrafo 7. En el mismo sentido, Eide, A. Non-citizens and minority rights, report of the European Commission for Democracy through Law, CDL(2006)055, párrafo 2. 


\section{Los artículos 2.1 y 26 del Pacto}

El Pacto prohíbe en su artículo 2.1 la discriminación en el goce de los derechos en él reconocidos, y consagra en su artículo 26 la igualdad ante la ley, la igual protección de la ley, la prohibición de toda discriminación bajo la ley y la garantía de efectiva protección contra cualquier discriminación ${ }^{28}$. Los derechos reconocidos por estos dos artículos tienen carácter universal, y pueden también ser invocados por las minorías ${ }^{29}$.

El concepto de discriminación es generalmente utilizado en derecho internacional para hacer referencia a una distinción irrazonable, no justificada o arbitraria que implica negar la igualdad de trato a un individuo en relación con otros individuos en razón de la pertenencia del primero a una determinada categoría de personas ${ }^{30}$. Corresponde a quienes afirmen que la distinción es legítima probar tal extremo ${ }^{31}$.

El Comité de Derechos Humanos ha definido la discriminación prohibida por el Pacto como cualquier distinción, exclusión, restricción o preferencia que está basada en la raza, en el color, en el sexo, en la lengua, en la religión, en criterios políticos o de opinión, en el origen nacional o social, en la propiedad, en el nacimiento o en otro estatus, y que tiene el propósito o el efecto de anular o de impedir el reconocimiento, el goce o el ejercicio de todas las personas, en pie de igualdad, de todos los derechos y libertades ${ }^{32}$. Se trata pues de una enumeración abierta de las bases de discriminación prohibidas $^{33}$.

Por supuesto que la prohibición de discriminación no obliga a dar a todos un tratamiento idéntico en toda circunstancia ${ }^{34}$. En efecto, tan discriminatorio puede resultar tratar de modo diferente dos circunstancias o personas iguales, como tratar de modo igual dos circunstancias o personas diferentes. Por ello es que no hay discriminación si el criterio de distinción aplicado es razonable y objetivo, y existe una finalidad legítima ${ }^{35}$.

28 General Comment No. 18: Non-discrimination, HRI/GEN/1/Rev.1 at 26 (1994), párrafo 1. La Declaración se refiere a la obligación de los Estados de adoptar medidas para que "las personas pertenecientes a minorías puedan ejercer plena y eficazmente todos sus derechos humanos y libertades fundamentales" (artículo 4.1) y para que "las personas pertenecientes a minorías puedan participar plenamente en el progreso y el desarrollo económicos de su país" (artículo 4.5).

29 General Comment No. 23: The rights of minorities (Art. 27), párrafo 4.

30 Ver el trabajo clásico de Mckean, W. Equality and discrimination under International Law, Oxford: Clarendon Press, 1985. Ver también Lerner, N. Group rights and discrimination in international law. The Hague: Martinus Nijhoff Publishers, 2003.

31 Dissenting Opinion of Judge Tanaka, South West Africa cases (2nd phase), ICJ Reports 1966, pp. 284316.

32 General Comment No. 18: Non-discrimination, párrafo 7.

33 Henrard, K. Equal Rights versus Special Rights? Minority Protection and the Prohibition of Discrimination. Luxemburg: European Commission, 2007, p. 37. Ver también Henrard, K. The Protection of Minorities through the Equality Provisions in the UN Human Rights Treaties: The UN Treaty Bodies. International Journal of Minority and Group Rights, 2007, 14: 141-180.

34 General Comment No. 18: Non-discrimination, párrafo 8. Ver Tomuschat, C. Equality and NonDiscrimination under the CCPR. Festschrift für Hans-Jûrgen Schlochauer. Berlin: Walter de Gruyter, 1981, pp. 712-716.

35 General Comment No. 18: Non-discrimination, párrafo 13. 
Al hacer referencia a las distinciones que tengan el propósito o el efecto de afectar los derechos la definición incorpora la consideración tanto de las discriminaciones directas (hay un objetivo discriminatorio) como de las discriminaciones indirectas (no hay un objetivo pero sí un efecto discriminatorio). Aunque el Comité fue inicialmente reticente a incluir los supuestos de discriminación indirecta como casos de violación del Pacto, en los casos más recientes el criterio ha sido aceptado con mayor facilidad ${ }^{36}$. Por otra parte, el Comité reconoce que la discriminación no siempre está basada en una distinción legalmente establecida, sino que a veces puede resultar de las circunstancias fácticas ${ }^{37}$. De este modo se incorpora la consideración tanto de las discriminaciones de iure como de las discriminaciones de facto.

El artículo 2.1 y el artículo 26 del Pacto no son una mera repetición del mismo derecho $^{38}$. El artículo 2.1 contiene un principio general de no discriminación en los derechos del Pacto $^{39}$, de modo que ha de leerse de forma complementaria a los artículos que establecen los derechos sustantivos. El artículo 26, por su parte, contiene un derecho autónomo a la igualdad ante la ley y la igual protección de la ley sin discriminación, así como la garantía de la protección efectiva frente a cualquier discriminación de iure o de facto en cualquier campo regido por una autoridad estatal ${ }^{40}$.

Los criterios de discriminación que tienen mayor interés en relación con las minorías son los de etnia, religión y lengua. Sobre estos dos últimos hay varios precedentes en el Comité de Derechos Humanos. En cuanto a la discriminación religiosa, el Comité declaró en Waldman v. Canadá que existía una violación del artículo 26 en la decisión estatal de financiar sólo las escuelas católicas y no las de otras religiones ${ }^{41}$. Algo semejante sucedió en Sister Immaculate v. Sri Lanka, en el que se halló una violación del artículo 26 y de la libertad religiosa del artículo 18 en una negativa injustificada a otorgar reconocimiento legal a una orden religiosa católica ${ }^{42}$. Sin embargo, si la

36 Barelli, M.. et. al. Minority groups and litigation: A review of developments in international and regional jurisprudence, p. 7. Henrard, K. Equal Rights versus Special Rights? Minority Protection and the Prohibition of Discrimination, p. 40-41.

37 General Comment No. 18: Non-discrimination, párrafo 9.

38 Morawa, A. The United Nations treaty monitoring bodies and minority Rights, with particular emphasis on the Human Rights Committee. Mechanisms for the Implementation of Minority Rights. Germany: Council of Europe Publishing, 2004, p. 39. Petričušić, A. The Rights of Minorities in International Law: Tracing Developments in Normative Arrangements of International Organizations, p. 7. Morawa, A. The Concept of Non-Discrimination: An Introductory Comment. In Journal of Ethnopolitics and Minorities in Europe [interactive]. 2002, 3: 1-2 [visitado 06/06/2013]. http://www.ecmi.de/jemie/download/Focus3-2002_ Morawa.pdf.

39 General Comment No. 18: Non-discrimination, párrafo 12.

40 General Comment No. 18: Non-discrimination, párrafo 12.

41 Arieh Hollis Waldman (Initially represented by Mr. Raj Anand from Scott \& Aylen, a law firm in Toronto, Ontario) v. Canada, communication 694/1996, CCPR/C/67/D/694/1996 (1999).

42 Sister Immaculate Joseph and 80 Teaching Sisters of the Holy Cross of the Third Order of Saint Francis in Menzingen of Sri Lanka v. Sri Lanka, communication 1249/2004, CCPR/C/85/D/1249/2004 (2005). Un caso parecido (negativa al reconocimiento legal con un determinado estatus) fue tratado en Sergei Malakhovsky and Alexander Pikul v. Belarus, communication 1207/2003, CCPR/C/84/D/1207/2003 (2005). Aquí se halló una violación del artículo 18, sin analizar el caso a la luz del artículo 22 (como pedían los denunciantes) ni del 26. 
distinción es objetiva y razonable no existirá violación del artículo 26, como sucedió en el caso Karnel Singh Bhinder v. Canada, en el que se declaró legítima la obligación de utilizar un casco impuesta a un obrero sikh (cuya religión manda cubrirse la cabeza con un turbante $)^{43}$.

Respecto de la discriminación en razón de la lengua, en el caso J. G. A. Diergaardt v. Namibia relativo a la comunidad Baster en este país ${ }^{44}$, se entendió que la prohibición que se había impuesto a la administración pública de utilizar la lengua afrikáans constituía un trato discriminatorio hacia los miembros de la comunidad Baster en los términos del artículo 26 del Pacto. En Antonina Ignatane v. Letonia se declaró la violación del artículo 25 (derecho a participar en la vida pública) en relación con el artículo 2 en cuanto prohíbe la discriminación en razón del idioma, por la exclusión de una candidata a las elecciones municipales perteneciente a la minoría de origen ruso ${ }^{45}$. En cambio, en Ballantyne, Davidson, McIntyre v. Canadá se declaró que no había discriminación de los angloparlantes en el sentido del artículo 26, aunque sí violación de la libertad de expresión del artículo 19, en relación con la norma que establecía que en Quebec sólo podían realizarse anuncios publicitarios en francés ${ }^{46}$. Finalmente, en el caso Dominique Guesdon v. France se declaró que no existía violación del principio de igualdad ante la ley del artículo 26 por el hecho de que el Estado no había suministrado un intérprete a un bretón que prefería expresarse en su lengua materna y no en francés, a pesar de que hablaba este último idioma ${ }^{47}$.

Para que exista violación del artículo 26 o del artículo 2 es necesario que se demuestre la existencia de un concreto acto discriminatorio. Así, en el caso Jarle Jonassen v. Noruega el Comité analizó la comunicación de un grupo de miembros de la comunidad Sami dedicados a la cría de renos que alegaba que una sentencia judicial que les había privado de la propiedad de sus tierras se había basado en otra sentencia de fines del siglo XIX, época en la que los Sami eran manifiestamente discriminados. El Comité sin embargo declaró la comunicación inadmisible porque entendió que los denunciantes no habían demostrado concretamente de qué modo la sentencia reciente resultaba discriminatoria ${ }^{48}$.

Interesa enfatizar la diferencia entre el principio de igualdad y no discriminación y la protección de las minorías tal como surge del artículo 27 del Pacto. Mientras que el principio de igualdad y no discriminación asegura que no haya diferencias injustificadas de tratamiento entre las personas, el artículo 27 exige por el contrario que, bajo ciertas condiciones, se reconozcan diferencias de tratamiento para proteger y promover las formas de vida de las minorías. Dado que los artículos 2.1 y 26 pueden ser invocados

43 Karnel Singh Bhinder v. Canada, communication 208/1986, CCPR/C/37/D/208/1986 (1989).

44 J.G.A. Diergaardt (late Captain of the Rehoboth Baster Community) et al. v. Namibia, communication 760/1997, CCPR/C/69/D/760/1997 (2000).

45 Antonina Ignatane v. Latvia, communication 884/1999, CCPR/C/72/D/884/1999 (2001).

46 Ballantyne, Davidson, McIntyre v. Canada, communications 359/1989 and 385/1989, CCPR/ C/47/D/359/1989 and 385/1989/Rev.1 (1993).

47 Dominique Guesdon v. France, communication 219/1986, CCPR/C/39/D/219/1986 (1990).

48 Jarle Jonassen and members of the Riast/Hylling reindeer herding district $v$. Norway. 
por cualquiera, también los miembros de las minorías pueden beneficiarse de ellos. En cambio, el artículo 27 está previsto sólo para los miembros de grupos minoritarios.

De este modo, las minorías gozan de una doble protección. Por un lado, la protección proveniente del principio de igualdad y no discriminación. Por otro lado, la protección específica para sus formas de vida del artículo 27. Se trata de dos mecanismos independientes. Así, por ejemplo, en el ya citado caso J. G. A. Diergaardt ${ }^{49}$, se entendió que la prohibición de utilizar la lengua afrikáans constituía un trato discriminatorio hacia los miembros de la comunidad Baster en los términos del artículo 26, pero no violaba sus derechos bajo el artículo 27.

Del mismo modo que respecto del artículo 27 , la protección de que puedan gozar las minorías por acción de los artículos 2 y 26 se extiende también a los extranjeros ${ }^{50}$.

\section{Las medidas positivas}

Las disposiciones que hemos venido mencionando (artículos 2.1, 26 y 27 del Pacto), están redactadas en términos amplios. Esas disposiciones exigen, como mínimo, una abstención de parte del Estado. Por ejemplo, el derecho de los miembros de una minoría a participar de la vida religiosa del grupo exige que el Estado no impida esa participación. En cuanto se satisfacen mediante acciones negativas del Estado los derechos específicos de las minorías no se diferencian de los derechos universales de los que, como ya sabemos, también gozan éstas. Así, en la medida en que implique una abstención de parte del Estado no hay diferencia entre la libertad religiosa del artículo 18 del Pacto y el derecho a participar en la vida religiosa del grupo del artículo 27.

Sin embargo, en muchos casos esas provisiones no se satisfacen sólo mediante acciones negativas del Estado, sino que exigen también su intervención activa ${ }^{51}$. Según el artículo 2 del Pacto los Estados están obligados a "respetar y garantizar" los derechos allí recogidos, así como a adoptar "las disposiciones legislativas o de otro carácter que fueren necesarias" para hacer efectivos esos mismos derechos. La misma obligación surge del artículo 1.2 de la Declaración. Aunque los Estados disponen de una cierta latitud para decidir cuáles son las medidas más apropiadas para hacer efectivos los derechos reconocidos en el Pacto ${ }^{52}$, están obligados a adoptarlas.

Es posible afirmar, en el contexto del artículo 27 del Pacto, que para garantizar los derechos reconocidos a los individuos pertenecientes a grupos minoritarios es necesario que el Estado adopte, en algunos casos, medidas que pueden ser calificadas como medidas positivas. Así lo ha hecho, en efecto, el Comité de Derechos Humanos

49 J.G.A. Diergaardt (late Captain of the Rehoboth Baster Community) et al. v. Namibia.

50 General Comment No. 15: The position of aliens under the Covenant, párrafos 2 y 7.

51 Ver el comentario de Akermark, A. Justifications of Minority Protection in International Law. London: Kluwer, 1997, p. 139. Ver también Capotorti, F. Study of the Rights of Persons belonging to Ethnic, Religious and Linguistic Minorities, p. 36.

52 General Comment No. 18: Non-discrimination, párrafo 4. General Comment No. 03: Implementation at the national level (Art. 2), HRI/GEN/1/Rev.6 at 125, párrafo 1. 
tanto en su Comentario general ${ }^{53}$ sobre el artículo 27 como en su respuesta a diversas comunicaciones en las que particulares denunciaban la violación de ese artículo ${ }^{54}$. Algo semejante sucede en el contexto de la Declaración. El artículo 4 impone a los Estados el deber de adoptar diferentes medidas para "crear condiciones favorables a fin de que las personas pertenecientes a minorías puedan expresar sus características y desarrollar su cultura, idioma, religión, tradiciones y costumbres" (artículo 4.2); para que "las personas pertenecientes a minorías puedan tener oportunidades adecuadas de aprender su idioma materno o de recibir instrucción en su idioma materno" (artículo 4.3); para "promover el conocimiento de la historia, las tradiciones, el idioma y la cultura de las minorías que existen en su territorio", así como para que las personas pertenecientes a minorías puedan "adquirir conocimientos sobre la sociedad en su conjunto" (artículo 4.4).

El primer artículo de la Declaración, además, afirma que los Estados deben "fomentar las condiciones para la promoción" de la identidad de las minorías (artículo 1.1), para lo que es necesario recurrir a "medidas apropiadas, legislativas y de otro tipo" (artículo 1.2). Según el comentario del Grupo de Trabajo sobre las Minorías, la protección de la identidad significa no sólo que el Estado debe abstenerse de políticas que tengan el propósito o el efecto de asimilar a las minorías en la cultura dominante, sino que también debe protegerla de las actividades de terceros que tengan un efecto asimilatorio ${ }^{55}$.

Las medidas positivas han de permitir por un lado, que los miembros de la minoría puedan expresar las características tradicionales del grupo (lo que puede incluir el derecho a usar vestimentas tradicionales y a ganarse la vida de acuerdo con sus propias formas culturales); y, por otro lado, que los individuos, en comunidad con otras personas pertenecientes al grupo, puedan desarrollar su cultura, lengua y tradiciones ${ }^{56}$.

Las medidas positivas pueden ser necesarias no sólo en relación con la protección y promoción de la identidad propia de las minorías, sino también en relación con el principio de igualdad y no discriminación ${ }^{57}$. Dado que los Estados están obligados a garantizar que los individuos bajo su jurisdicción gocen de los derechos emergentes del Pacto, puede ser necesario que éstos ejecuten medidas positivas para corregir desigualdades a las que están sujetas las minorías ${ }^{58}$. Así, en un Estado en el que las condiciones generales de una parte de la población le impiden el pleno goce de los derechos humanos, el Estado debe ejecutar acciones específicas para corregir esas condiciones. Estas acciones pueden significar otorgar, por un cierto tiempo, un trato preferencial a ciertas personas en relación con ciertas materias ${ }^{59}$.

53 General Comment No. 23: The rights of minorities (Art. 27), párrafos 6.1, 6.2, 7 y 9.

54 Ángela Poma Poma v. Peru, communication 1457/2006, CCPR/C/95/D/1457/2006, párrafo 7.2. Apirana Mahuika et al. v. New Zealand, párrafo 9.6.

55 Commentary on the United Nations Minorities Declaration, párrafo 28.

56 Commentary on the United Nations Minorities Declaration, párrafo 56.

57 Morawa, A. The Concept of Non-Discrimination: An Introductory Comment, p. 7.

58 Minorities Rights: International Standards and Guidance for Implementation, United Nations High Commissioner of Human Rights, United Nations: New York, 2010, p. 15-16.

59 General Comment No. 18: Non-discrimination, párrafo 10. 
Las medidas positivas adoptadas para garantizar la igualdad real reciben el nombre de acciones afirmativas o medidas especiales ${ }^{60}$. Aunque aquí nos interesan estas medidas en relación con las minorías, ellas son aplicables a cualquier grupo desaventajado por su raza, origen étnico, lengua, etc. De hecho las acciones afirmativas están previstas en otros instrumentos internacionales como forma de protección de grupos desaventajados. Así ocurre en el caso de la Convención Internacional para la Eliminación de Todas las Formas de Discriminación Racial ${ }^{61}$ y de la Convención Internacional para la Eliminación de Todas las Formas de Discriminación contra la Mujer ${ }^{62}$.

Como explican Thornberry y Eide, la acción afirmativa no es en sí misma contraria al Pacto Internacional de Derechos Civiles y Políticos ${ }^{63}$. En efecto, el principio de no discriminación no prohíbe cualquier distinción entre las personas, sino sólo aquellas que no sean objetivas y razonables. Otros autores, como Bayefsky y Doebbler, han sostenido que la acción afirmativa puede ser incluso obligatoria en determinadas circunstancias ${ }^{64}$.

Existen dos límites fundamentales a estas medidas: ellas no deben llevar a situaciones discriminatorias y deben tener una duración determinada. En efecto, las acciones afirmativas pueden chocar con la prohibición de discriminación si no están correctamente encuadradas. Como explica Bossuyt, existe una tensión inherente entre ambas: mientras que la prohibición de discriminación remueve factores como la raza, el género, la nacionalidad, etc. en la aplicación de las normas jurídicas, la acción afirmativa obliga a tener en cuenta tales factores. La sola constatación de que el criterio utilizado para la acción afirmativa es aquel respecto del cual se ha producido una situación histórica de desventaja (por ejemplo, la pertenencia a una minoría nacional) no es suficiente para legitimar la acción afirmativa a la luz del principio de no discriminación. Ha de evaluarse respecto de esa acción afirmativa la concreta vinculación del criterio de distinción y del derecho involucrado ${ }^{65}$.

60 La primera mención a estas medidas puede encontrarse en las observaciones del gobierno indio durante la elaboración del Pacto Internacional sobre Derechos Económicos, Sociales y Culturales (A/C.3/SR.1182, párrafo 17) y luego durante la elaboración del Pacto Internacional de Derechos Civiles y Políticos (A/C.3/ SR.1257, párrafo 18; A/C.3/SR.1258, paras. 18, 38, 45, 47). El objetivo era incluir un párrafo en el Pacto que permitiera expresamente las medidas tomadas para proteger un sector determinado de la población, desfavorecido en razón de determinadas circunstancias históricas, para restablecer las condiciones de igualdad y por el tiempo limitado que fuera necesario para alcanzar este fin. Ver Bossuyt, M. Guide to the "Travaux Préparatoires" of the International Covenant on Civil and Political Rights. Dordrecht: Martinus Nijhoff Publishers, 1987, p. 55.

61 Artículo 1.4 de la Convención Internacional sobre la Eliminación de todas las Formas de Discriminación Racial (adoptada 21/12/1965, en vigor desde 04/01/1969), UNTS 660-195.

62 Artículo 4 de la Convención sobre la Eliminación de todas las Formas de Discriminación contra la Mujer (adoptada 18/12/1979, en vigor desde 03/09/1981), UNTS 1249-13.

63 P. Thornberry. International Law and the Rights of Minorities, p. 284. Eide, A. Protection of minorities: possible ways and means of facilitating the peaceful and constructive solution of problems involving minorities, párrafo 172 .

64 Bayefsky, A. F. The principle of equality or non-discrimination in international law. Human Rights Law Journal, 1990, 11(1): 27. Doebbler, C. F. J. Principle of Non-Discrimination in International Law. Washington: CD Publishing, 1995, p. 13.

65 Bossuyt, M. The concept and practice of affirmative action. Preliminary report submitted by Mr. Marc Bossuyt, Special Rapporteur, in accordance with Sub-Commission resolution 1998/5, E/CN.4/ Sub.2/2000/11, párrafos 41-44 y 62 . 
El segundo requisito para la legitimidad de las acciones afirmativas es que tengan una duración limitada. Las acciones afirmativas no pueden llevar a la permanencia de estándares separados para diferentes grupos, y no pueden prolongarse luego de que han sido alcanzados sus objetivos ${ }^{66}$.

Las medidas positivas tomadas al amparo del artículo 27 tienen una finalidad diferente de las adoptadas bajo el principio de no discriminación. El objetivo de las segundas es el de garantizar la igualdad real de los individuos. Las primeras, en cambio, buscan proteger el derecho de las personas a ser diferentes. Por ello es que las medidas positivas adoptadas para proteger los modos de vida de las minorías no están sometidas al límite temporal ${ }^{67}$.

Sin embargo, sí están sometidas a la regla de que ellas mismas no violen derechos de terceros. Por ejemplo, en el ya nombrado caso Ballantyne, Davidson, McIntyre v. Canadá el Comité declaró que la prohibición de usar un idioma distinto del francés en las publicidades comerciales violaba la libertad de expresión del artículo 19 (aunque no era discriminatoria en los términos del artículo 26 ni violaba el artículo 27 porque aunque los angloparlantes son minoritarios en la provincia francófona de Quebec no son una minoría en los términos de este último artículo) ${ }^{68}$.

Un aspecto que resulta de interés en relación con las medidas positivas es el de la situación de los extranjeros. Como ya hemos dicho, la protección del artículo 27, así como la de los artículos 2 y 26, se extiende también a los extranjeros. Sin embargo, tratándose de medidas que impliquen una actividad positiva de parte del Estado, pueden existir algunos matices. La adopción de medidas positivas no es en sí misma obligatoria, de modo que si el Estado no está obligado a adoptar estas acciones podría también excluir del ámbito de protección a los extranjeros. La cuestión deja entonces de ser un asunto de todo o nada, para volverse un asunto a analizar respecto de cada derecho en particular $^{69}$. La exclusión, por supuesto, debería estar basada en criterios razonables y objetivos. Como apunta acertadamente Henrard, la diferencia de trato en base a la nacionalidad podría llevar a una situación de discriminación indirecta prohibida sobre otras bases, como por ejemplo la raza ${ }^{70}$.

\section{Conclusiones}

El objetivo fundamental de este trabajo ha sido identificar las diferentes categorías de derechos que corresponden a las minorías en el sistema del Pacto. La protección de las minorías, en el sentido amplio del término, abarca dos mecanismos: la protección de

66 Bossuyt, M., The concept and practice of affirmative action, párrafo 66.

67 Lerner, N. Group rights and discrimination in international law, p. 40.

68 Ballantyne, Davidson, McIntyre v. Canada.

69 Eide, A. Non-citizens and minority rights, párrafo 14.

70 Henrard, K. Equal Rights versus Special Rights? Minority Protection and the Prohibition of Discrimination, p. 38. 
la identidad propia de éstas mediante el artículo 27 y la garantía de la igualdad formal y material mediante los artículos 2.1 y 26.

El artículo 27 del Pacto Internacional de Derechos Civiles y Políticos reconoce derechos específicos de las minorías en general. Sin embargo, la implementación concreta de este artículo mediante medidas positivas puede dar nacimiento a derechos específicos de minorías determinadas. Los derechos reconocidos por el artículo 27, aunque tengan por finalidad la protección de grupos, son derechos de titularidad individual.

Los artículos 2.1 y 26 del Pacto, por su parte, se refieren al principio de no discriminación. Este principio tiene carácter universal, y puede también ser invocado por las minorías. Al igual que sucede en el caso anterior, la implementación concreta de este artículo mediante medidas positivas puede dar nacimiento a derechos específicos de minorías determinadas.

Las medidas positivas destinadas a implementar los derechos reconocidos en términos amplios en el Pacto pueden entrar en tensión entre sí. En efecto, las medidas adoptadas en el contexto del artículo 27 podrían vulnerar derechos de terceros o resultar discriminatorias a su respecto. Las medidas positivas encaminadas a la igualdad, por su parte, no deberían significar políticas indiscriminadamente uniformizantes que perjudicaran la finalidad protectora del artículo 27 .

\section{Referencias}

Akermark, A. Justifications of Minority Protection in International Law. London: Kluwer, 1997.

Andrysek, O. Report on the Definition of Minorities. Netherlands: Netherlands Institute of Human Rights, 1986.

Barelli, M.. et. al. Minority groups and litigation: A review of developments in international and regional jurisprudence. Liverpool: Minority Rights Group International, 2011.

Bayefsky, A. F. The principle of equality or non-discrimination in international law. Human Rights Law Journal, 1990, 11(1): $1-34$.

Bengoa, J. Existence and recognition of minorities, working paper for the Working Group of Minorities, E/CN.4/Sub.2/ AC.5/2000/WP.2.

Bisaz, C. The Concept of Group Rights in International Law. Leiden: Martinus Nijhoff Publishers, 2012.
Bossuyt, M. Guide to the "Travaux Préparatoires" of the International Covenant on Civil and Political Rights. Dordrecht: Martinus Nijhoff Publishers, 1987, p. 55.

Bossuyt, M. The United Nations and the Definition of Minorities. Plural Societes. 1990, 21: 129-136.

Bossuyt, M. The concept and practice of affirmative action. Preliminary report submitted by Mr. Marc Bossuyt, Special Rapporteur, in accordance with SubCommission resolution 1998/5, E/CN.4/ Sub.2/2000/11.

Capotorti, F. Study of the Rights of Persons belonging to Ethnic, Religious and Linguistic Minorities. New York: United Nations, 1991, p. 36.

Díaz Pérez de Madrid, A. La protección de las minorías en Derecho internacional. Granada: Editorial Universidad de Granada, 2004.

Doebbler, C. F. J. Principle of NonDiscrimination in International Law. Washington: CD Publishing, 1995. 
Eide, A. Non-citizens and minority rights, report of the European Commission for Democracy through Law, CDL(2006)055.

Eide, A. Protection of minorities: possible ways and means of facilitating the peaceful and constructive solution of problems involving minorities, E/CN.4/Sub.2/1993/34.

Ermacora, F. The protection of minorities before the United Nations, Recueil des Cours 182. Leiden: Martinus Nijhoff Publishers, 1983.

González Hidalgo, E.; Ruiz Vieytez, E. La definición implícita del concepto de minoría nacional en el Derecho Internacional. Derechos y libertades: Revista del Instituto Bartolomé de las Casas. 2012, 27: 17-56.

Henrard, K. Devising an Adequate System of Minority Protection. Individual Human Rights, Minority Rights and the Right to Self-Determination. The Hague: Martinus Nijhoff Publishers, 2000.

Henrard, K. Equal Rights versus Special Rights? Minority Protection and the Prohibition of Discrimination. Luxemburg: European Commission, 2007.

Henrard, K. Minorities, International Protection. Max Planck Encyclopedia of Public International Law [interactivo]. 2010 [visitado 20/08/2013]. http://www.mpepil. com.

Henrard, K. The Interrelationship between Individual Human Rights, Minority Rights and the Right to Self-Determination and Its Importance for the Adequate Protection of Linguistic Minorities. The Global Review of Ethnopolitics. 2001, 1(1): 41-61.

Henrard, K. The Protection of Minorities through the Equality Provisions in the UN Human Rights Treaties: The UN Treaty Bodies. International Journal of Minority and Group Rights, 2007, 14: 141-180.

Kymlicka, W. The Internationalization of Minority Rights. International Journal of Constitutional Law, 2008, 6(1): 1-32.

Lerner, N. Group rights and discrimination in international law. The Hague: Martinus Nijhoff Publishers, 2003.

Lerner, N. Las Naciones Unidas y las minorías: a propósito de la Declaración de la Asamblea General de 18 de diciembre de 1992. Revista española de derecho internacional. 1993, 45(2): 269-282.
Lerner, N. The Evolution of Minority Rights in International Law. Peoples and Minorities in International Law. London: Martinus Nijhoff Publishers, 1993.

Meijknecht, A. Towards International Personality: The Position of Minorities and Indigenous Peoples in International Law. Antwerpen: Intersentia, 2001, p. 136.

Morawa, A. The Concept of NonDiscrimination: An Introductory Comment. In Journal of Ethnopolitics and Minorities in Europe [interactive]. 2002, 3: 1-2 [visitado 06/06/2013]. http://www.ecmi.de/jemie/ download/Focus3-2002_Morawa.pdf.

Morawa, A. The United Nations treaty monitoring bodies and minority Rights, with particular emphasis on the Human Rights Committee. Mechanisms for the Implementation of Minority Rights. Germany: Council of Europe Publishing, 2004, p. 29-53.

Pentassuglia, G. Minority Groups and Judicial Discourse in International Law. The Hague: Martinus Nijhoff Publishers, 2009.

Pentassuglia, G. Minority Rights, Human Rights: A review of basic concepts, entitlements and implementation procedures under International law. Mechanisms for the implementation of minority rights, Berlin: Council of Europe Publishing, 2004, p. 9-28.

Pentassuglia, G., Minorities in international law: an introductory study. Strasbourg: Council of Europe Publications, 2002.

Petričušić, A. The Rights of Minorities in International Law: Tracing Developments in Normative Arrangements of International Organizations. Croatian International Relations Review, 2005, XI(38/39): 7.

Ramaga, P. The Group Concept in Minority Protection. Human Rights Quarterly. 1993, 15(3): 575-588.

Rodley, N. Conceptual Problems in the Protection of Minorities: International Legal Development. Human Rights Quarterly. 1995, 17(1): 48-71.

Rouland, N.; Pierré-Caps, S.; Poumarède, J. Droit des minorités et des peuples autochtones. Paris: Presses Universitaires de France, 1996. 
Thio, L. Managing Babel: The International Legal Protection of Minorities in the Twentieth Century. The Hague: Martinus Nijhoff Publishers, 2005.

Thornberry, P. International Law and the Rights of Minorities. Oxford: Clarendon Press, 1991.

Tomuschat, C. Equality and NonDiscrimination under the CCPR. Festschrift für Hans-Jûrgen Schlochauer. Berlin: Walter de Gruyter, 1981, pp. 691-716.

Vijapur, A. International protection of minority rights. International Studies, 2006, 43(4): 367-394.

\section{Human Rights Committee}

General Comment No. 03: Implementation at the national level (Art. 2), HRI/GEN/1/ Rev.6 at 125.

General Comment No. 15: The position of aliens under the Covenant, HRI/GEN/1/ Rev.1 at 18.

General Comment No. 18: Non-discrimination, HRI/GEN/1/Rev.1 at 26.

General Comment No. 23: The rights of minorities (Art. 27), CCPR/C/21/Rev.1/ Add.5.

Ángela Poma Poma v. Peru, communication 1457/2006, CCPR/C/95/D/1457/2006.

Antonina Ignatane $v$. Latvia, communication 884/1999, CCPR/C/72/D/884/1999 (2001).

Apirana Mahuika et al. v. Nueva Zelanda, communication 547/1993, CCPR/ C/70/D/547/1993 (2000).

Arieh Hollis Waldman (Initially represented by Mr. Raj Anand from Scott \& Aylen, a law firm in Toronto, Ontario) v. Canada, communication 694/1996, CCPR/ C/67/D/694/1996 (1999).

Ballantyne, Davidson, McIntyre v. Canada, communications 359/1989 and 385/1989, CCPR/C/47/D/359/1989 and 385/1989/ Rev.1 (1993).

Bernard Ominayak Chef of the Lubicon Lake Band v. Canada, communication 167/1984, Supp. 40 (A/45/40) at 1 (1990).

George Howard v. Canada, communication 879/1999, CCPR/C/84/D/879/1999 (2005).

Ilmari Lansman et al. v. Finlandia, communication 511/1992, CCPR/C/52/D/ 511/1992 (1994).
Ivan Kitok v. Sweden, communication 197/1985, CCPR/C/33/D/197/1985 (1988).

J.G.A. Diergaardt (late Captain of the Rehoboth Baster Community) et al. $v$. Namibia, communication 760/1997, CCPR/ C/69/D/760/1997 (2000).

Jarle Jonassen and members of the Riast/ Hylling reindeer herding district $v$. Norway, communication 942/2000, CCPR/ C/76/D/881/1999 (2002).

Jouni E.Länsmanetal.v. Finland, communication 671/1996, CCPR/C/58/D/671/1995 (1996).

Jouni Länsman, Eino Länsman and the Muotkatunturi Herdsmen's Committee v. Finland communication 1023/2001, CCPR/ C/83/D/1023/2001.

Karnel Singh Bhinderv. Canada, communication 208/1986, CCPR/C/37/D/208/1986 (1989).

Sandra Lovelace v. Canada, communication R.6/24, Supp. No. 40 (A/36/40) at 166 (1981).

Sergei Malakhovsky and Alexander Pikul v. Belarus, communication 1207/2003, CCPR/ C/84/D/1207/2003 (2005).

Sister Immaculate Joseph and 80 Teaching Sisters of the Holy Cross of the Third Order of Saint Francis in Menzingen of Sri Lanka v. Sri Lanka, communication 1249/2004, CCPR/C/85/D/1249/2004 (2005).

\section{Permanent Court of International Justice} e International Court of Justice

Rights of Minorities in Upper Silesia (Minority Schools), Germany v. Poland (Judgment), 1928 PCIJ Series A, 15.

Access to German minority schools in Upper Silesia (Advisory Opinion), 1931 PCIJ Series $\mathrm{A} / \mathrm{B}, 40$.

Minority Schools in Albania (Advisory Opinion), 1935 PCIJ Series A/B, 64.

South West Africa cases (2nd phase), ICJ Reports 1966.

\section{Otros documentos institucionales}

Commentary on the United Nations Minorities Declaration, Working Group on Minorities, E/CN.4/Sub.2/AC.5/2005/2.

Minorities Rights: International Standards and Guidance for Implementation, United Nations High Commissioner of Human Rights, United Nations: New York, 2010, p. 15-16. 


\title{
MAŽUMŲ TEISĖS TARPTAUTINIAME PILIETINIŲ IR POLITINIŲ TEISIŲ PAKTE: KONCEPTUALUS APTARIMAS
}

\author{
Fernando Arlettaz
}

Saragosos universitetas, Ispanija

Santrauka. Straipsnyje aptariamos mažumu teises pagal Tarptautini pilietiniu ir politiniu teisiu pakta.

Paktas įtvirtina konceptualu skirtuma tarp visuotiniu teisiu, specialiujų mažumu teisiu (taikomu visoms mažumoms) bei specialiujų konkrečiu mažumu teisiu. Visuotinès teisès taikomos visiems asmenims (pavyzdžiui, „niekas negali büti kankinamas") arba visiems tam tikros klases atstovams (pavyzdžiui, „visos šeimos turi teisę ¿̇ apsanga"). Mažumu grupes ir ju nariai šiomis teisemis gali naudotis kaip ir bet kurie kiti asmenys ar ju grupes. Specifinès mažzmu teisès (bendruoju požiūriu) yra suteikiamos visoms mažumu grupems (pavyzdžiui, „mažumu grupés turi teisę kalbèti savo gimtaja kalba") arba ju nariams (pavyzdžiui, "asmenys, priklausantys mažumoms, turi teisę kalbeti mažumos kalba"). Tam tikros mažumu grupès specifinès teisès yra suteikiamos konkrečioms grupèms pagal ju charakteristikas (pavyzdžiui, „kalbine mažuma X turi teise kalbetti X kalba“) arba ju nariams (pavyzdžiui, „bet koks kalbines mažumos $X$ narys turi teisę kalbeti X kalba").

Tarptautinèse sutartyse ir deklaracijose numatomos visuotinès teisés ir konkrečiu mažumu teisès apskritai. Tačiau šios teises paprastai pripažistamos plačiaja prasme, o ju igyvendinimas gali lemti specifiniu teisiu pripažinima tik tam tikroms mažumoms ar jų nariams.

Mažumu apsauga (plačiaja prasme) yra pagrissta dviem ramsčiais: pirmasis - apsauga nuo diskriminacijos, antrasis - mažumu teisiu apsauga siauraja prasme. Pirmasis ramstis yra bendrojo pobūdžio ir reikalauja pagarbos tiek formaliajai, tiek ir turinio lygybei. Mažumu teisiu siauraja prasme apsanga apima ypatinga ju tapatuma.

Lygybe formaliaja prasme yra igyvendinama per universaliasias teises tokia apimtimi, kiek jos suteikiamos mažumu grupems ir ju nariams. Norint pasiekti lygybę materialine prasme, kartais bütina taikyti skatinamąsias priemones. Igyvendinant sias priemones paprastai specifinès teisès suteikiamos mažumu grupems ar ju nariams. Skiriamuju mažumu savybiu apsauga taip pat igyvendinta per specifines teises.

Taigi, vienos teises skirtos apsaugoti mažumu gyvenimo büda (t. y. apsaugoti skirtumus), o kitomis siekiama, kad mažumoms bütu garantuota vienoda prieiga prie įvairiu socialiniu gerybiu (t. y. apsangoti lygybe). Kadangi pirmuoju aspektu siekiama apsaugoti ir skatinti mažumu kultüros ypatumus (pavyzdžiui, teise igyti išsilavinima gimtaja kalba), kitu aspektu siekiama iveikti sunkumus, su kuriais susiduria mažumu grupès ir jos nariai. Jie nera skirti skatinti ypatumus, bet nukreipti į sieki užtikrinti vienoda prieiga prie ištekliu ir vienodas socialines galimybes.

Tarptautinio pilietiniu ir politiniu teisiu pakto 27 straipsnis įtvirtina specialias mažumu teises, kuriomis siekiama apsaugoti ir skatinti ju gyvenimo formas. Nors ekonominès teisès nèra minimos (straipsnis įvardija tokias teises kaip „puoselèti savo kultūra, išpažinti ir praktikuoti savo religija, [ir] vartoti savo kalba"), jos gali büti išvedamos iš aiškiai apibrěžtu teisiu. Paktu pripažistamos teisès yra ne kolektyvinés, o individualios (",ose valstybèse, kuriose 
etninés, religinès ar kalbinès mažumos egzistuoja, asmenims, priklausantiems tokioms mažumoms, negali büti atimta teise [...]"). Teises, kurios pripažistamos Jungtiniu Tautu Generalinès Asamblëjos deklaracijoje dèl asmenu, priklausančiu tautinèms ar etninèms, religinèms ir kalbinèms mažumoms, teisiu, yra tokio paties pobüdžio.

Lygybès ir nediskriminavimo principas yra ìtvirtinti Pakto 2.1. ir 2.6. straipsniuose. Šis principas draudžia tiesiogine ir netiesiogine diskriminacija ir de iure, ir de facto. Kadangi tai universali teise, mažumos taip pat gali ja naudotis.

$\check{Z}$ mogaus teisiu komiteto nuomone, 27 straipsnis ipareigoja valstybes taikyti skatinančias priemones, kurios užkirstu kelia diskriminacijai. Specialios priemonès taip pat gali büti reikalingos, siekiant efektyvesnio lygybès ir nediskriminavimo principo igyvendinimo. Tačiau nei 27 straipsnis, nei 2.1. ar 26 straipsniai nenukreipiami individu, kurie priklauso specifinèms mažumoms, atžvilgiu. Konkretus šiu straipsniu igyvendinimas per skatinančias priemones galètu sukurti specifines teises žmonems, kurie priklauso tam tikrai mažumų grupei.

Reikšminiai žodžiai: mažumos, Tarptautinis pilietinių ir politiniu teisiu paktas, Žmogaus teisių komitetas, diskriminacija, skatinančios priemonès.

\section{MINORITY RIGHTS IN THE INTERNATIONAL COVENANT ON CIVIL AND POLITICAL RIGHTS: CONCEPTUAL CONSIDERATIONS}

\section{Fernando Arlettaz}

University of Zaragoza, Spain

Summary. The article discusses the rights of minorities in the system of the International Covenant on Civil and Political Rights.

It establishes a conceptual distinction between universal rights, specific rights of minorities in general and specific rights of particular minorities. Universal rights correspond to all individuals (e,g," "no one shall be subjected to torture") or all groups of a certain class (e.g., "all families are entitled to protection"). Minority groups and their members are entitled to these rights in the same way as any other individual or group. Specific rights of minorities in general are granted to minority groups (e.g., "minority groups have the right to speak their own language") or their members (e.g., "persons belonging to a minority group have the right to speak the language of the group"). Finally, specific rights of particular minorities are granted to specific groups identified by their characteristics (e.g., "the linguistic minority $X$ has the right to speak the X language") or their members (e.g., "any member of the linguistic minority $X$ has the right to speak the X language").

Treaties and international declarations often refer to universal rights and specific rights of minorities in general. However, these rights are usually recognised in broad terms, and their implementation may lead to the recognition of specific rights in favour of particular minorities or their members.

Protection of minorities (in the broad sense of the expression) is based on two pillars: on the one hand, the protection against discrimination; on the other hand, the protection 
of minorities in the strict sense. The first pillar is an aspect of the general principle of nondiscrimination and requires respect for both formal and substantive equalities. The protection of minorities in the strict sense involves the preservation of their particular identity.

The purpose of the equality in a formal sense is achieved through universal rights, to the extent that these are granted to minority groups and their members. On the contrary, to achieve equality in a substantive sense, it could be necessary to implement positive measures. The implementation of these measures will usually lead to the recognition of specific rights of minority groups or their members. Finally, the protection of the distinctive characteristics of the minorities will also be done through specific rights.

Thus, on the one hand, there are rights, which are intended to protect the minority forms of life (i.e. to protect differences), and, on the other hand, those that are intended to allow minorities equal access to different uniform social goods (i.e. to protect equality). Whereas the former ones seek to protect and promote the particularities of the minority culture (e.g., the right to receive education in their own language), the latter ones are recognised to overcome the disadvantages that minority groups and their members face and are not intended to promote peculiarities, but rather to permit equal access to resources and social opportunities.

Article 27 of the International Covenant on Civil and Political Rights enshrines specific rights of minorities intended to protect and promote their own forms of life. Although economic rights are not mentioned (the article refers to the rights "to enjoy their own culture, to profess and practise their own religion, [and] to use their own language"), they may stem from the explicitly recognised rights. The rights recognised by the Covenant are not collective, but individual rights ("in those States in which ethnic, religious or linguistic minorities exist, persons belonging to such minorities shall not be denied the right [...]"). The rights recognised in the Declaration of the United Nations General Assembly on the Rights of Persons Belonging to National or Ethnic, Religious and Linguistic Minorities are of the same nature.

The principle of equality and non-discrimination is contained in Articles 2.1 and 26 of the Covenant. This principle forbids direct and indirect discrimination, be it de iure or de facto. As this is a universal right, minorities can also benefit from it.

According to the Human Rights Committee, Article 27 imposes on States the adoption of positive measures that exceed an attitude of mere abstention. In the same way, positive measures may be necessary to make the principle of equality and non-discrimination effective. Although neither Article 27 nor Articles 2.1 and 26 refer to individuals belonging to a specific minority, the concrete implementation of these articles through positive measures may produce specific rights for persons belonging to particular minority groups.

Keywords: minorities, International Covenant on Civil and Political Rights, Committee on Human Rights, discrimination, positive measures.

Fernando Arlettaz, Saragosos universiteto Teisès sociologijos laboratorijos tyrèjas, socialinių mokslų (teisès krypties) daktaras. Mokslinių tyrimų kryptys: teisès sociologija, tarptautinè teisè, mažumų teisès, politinès teisès.

Fernando Arlettaz, University of Saragossa, Laboratory of Sociology of Law, Doctor of Social Sciences (Law), researcher. Research interests: law sociology, international law, minority rights, political rights. 